\title{
High resolution (3 Tesla) MRI-guided conformal brachytherapy for cervical cancer: consequences of different high-risk CTV sizes
}

\author{
James W. Anderson, PhD', Junyi Xia, PhD², Ryan T. Flynn, PhD², Joseph M. Modrick, PhD², Sudershan K. Bhatia, MD, MPH, PhD², \\ Geraldine M. Jacobson, MD, MPH, MBA3 , Yusung Kim, PhD² \\ IRadiation Oncology Department, Rush University, Chicago, 2Radiation Oncology Department, University of lowa, lowa City, ${ }^{3}$ Radiation \\ Oncology Department, West Virginia University, Morgantown, USA
}

\begin{abstract}
Purpose: To evaluate conventional brachytherapy (BT) plans using dose-volume parameters and high resolution (3 Tesla) MRI datasets, and to quantify dosimetric benefits and limitations when MRI-guided, conformal BT (MRIG-CBT) plans are generated.

Material and methods: Fifty-five clinical high-dose-rate BT plans from 14 cervical cancer patients were retrospectively studied. All conventional plans were created using MRI with titanium tandem-and-ovoid applicator (T\&O) for delivery. For each conventional plan, a MRIG-CBT plan was retrospectively generated using hybrid inverse optimization. Three categories of high risk (HR)-CTV were considered based on volume: non-bulky $(<20 \mathrm{cc})$, low-bulky $(>20 \mathrm{cc}$ and $<40 \mathrm{cc}$ ) and bulky $(\geq 40 \mathrm{cc})$. Dose-volume metrics of $\mathrm{D}_{90}$ of HR-CTV and $\mathrm{D}_{2 \mathrm{cc}}$ and $\mathrm{D}_{0.1 \mathrm{cc}}$ of rectum, bladder, and sigmoid colon were analyzed.

Results: Tumor coverage (HR-CTV $\mathrm{D}_{90}$ ) of the conventional plans was considerably affected by the HR-CTV size. Sixteen percent of the plans covered HR-CTV $\mathrm{D}_{90}$ with the prescription dose within $5 \%$. At least one OAR had $\mathrm{D}_{2 c c}$ values over the GEC-ESTRO recommended limits in $52.7 \%$ of the conventional plans. MRIG-CBT plans showed improved target coverage for $\mathrm{HR}-\mathrm{CTV} \mathrm{D}_{90}$ of 98 and $97 \%$ of the prescribed dose for non-bulky and low-bulky tumors, respectively. No MRIG-CBT plans surpassed the $\mathrm{D}_{2 \mathrm{cc}}$ limits of any OAR. Only small improvements $\left(\mathrm{D}_{90}\right.$ of $\left.80 \%\right)$ were found for large targets $(>40 \mathrm{cc})$ when using T\&O applicator approach.

Conclusions: MRIG-CBT plans displayed considerable improvement for tumor coverage and OAR sparing over conventional treatment. When the HR-CTV volume exceeded $40 \mathrm{cc}$, its improvements were diminished when using a conventional intracavitary applicator.
\end{abstract}

Key words: brachytherapy, cervical cancer, high-dose-rate brachytherapy, MRI-guided brachytherapy.

\section{Purpose}

Brachytherapy (BT) has played a central role in radiation therapy for cervical cancer since 1903. The standard of care for cervical cancer is a combination of external beam radiation therapy (EBRT) with concomitant cisplatinum-based chemotherapy and BT. The standard BT treatment technique has been unchanged for decades: 2D orthogonal radiographs are used to specify a dose to a fixed reference point, point A, relative to the intracavitary applicator. The patient anatomy and tumor location, not radiographically visible, are unused in the treatment planning process [1,2]. Recent advances in treatment planning based on 3D imaging enables the delineation of the target volumes and critical structures allowing volume-based inverse planning [3]. As magneticresonance imaging (MRI) provides superior soft tissue differentiation relative to computed tomography (CT) [4],
MRI-based treatment planning prior to each BT fraction is preferred. The patient's clinical target volume (CTV) and organs at risk (OARs) can be clearly identified and contoured on MRI datasets [4], allowing radiation delivery to macroscopic tumor volumes, and adaptive planning to account for tumor regression.

The American Brachytherapy Society (ABS) task group for cervical cancer [2] has adopted the Groupe Européen de Curiethérapie-European Society for Therapeutic Radiology and Oncology (GEC-ESTRO) recommendations for 3-D imaging based BT planning [5]. The planning method takes the pear-shaped isodose lines of conventional treatment and adjusts dwell times to optimize the minimum dose received by $90 \%, \mathrm{D}_{90}$, of the High Risk (HR)-CTV, while restricting the minimum dose to the hottest $2 \mathrm{cc}$ of each OAR, $\mathrm{D}_{2 \mathrm{cc}}$. Several studies [6-9] have investigated correlations between dose-volume-histogram (DVH) parameters from these op- 
timized treatments and conventional point doses from conventional planning. For example point $\mathrm{A}$ and International Commission on Radiation Units and measurements (ICRU) report \#38-defined rectum and bladder points [1] using pulsed-dose-rate (PDR) and high-dose-rate (HDR) BT. The goal is to understand the correlation between ICRU report \#38-defined points \& point $\mathrm{A}$ and DVH metrics [6-9]. One center, performing MRI-guided, conformal BT (MRIG-CBT) [10] reports that the mean HR-CTV volume for patients with local recurrence was significantly larger $(50 \pm 24 \mathrm{cc})$ than for patients without local recurrence $(34 \pm 23 \mathrm{cc}, p<0.05)$. Only $\mathrm{D}_{90}$ and $\mathrm{D}_{100}$ of the HR-CTV had a statistically significant effect on local tumor control, while the doses to intermediate-risk CTV and gross target volume did not [10]. This result implies that more analysis is needed into the dosimetric benefits and limitations of MRIG-CBT plans for different HR-CTV sizes. Previous studies considering HR-CTV size have only classified large and small volumes, and employed lower resolution MRI ( $<2$ Tesla) $[8,9]$. There is a need for improved contouring precision [4], accomplished with a high-resolution (3 Tesla) MRI [11]. Even though high-resolution (3 Tesla) MRI is being increas-

Table 1. Patient and plan characteristics

\begin{tabular}{|c|c|c|c|}
\hline Patient characteristics & & $N$ & $\%$ \\
\hline Patients & & 14 & \\
\hline \multicolumn{4}{|l|}{ FIGO stage } \\
\hline $\mathrm{IB}-\mathrm{IIB}$ & & 8 & 57 \\
\hline III-IV & & 6 & 43 \\
\hline \multicolumn{4}{|l|}{ Histopathology } \\
\hline Squamous cell carcinoma & & 11 & 79 \\
\hline Adenocarcinoma & & 2 & 14 \\
\hline Other & & 1 & 7 \\
\hline Lymph node involvement & & 4 & 29 \\
\hline 3 BT fractions (5.3-7.5 Gy) & & 5 & 36 \\
\hline 4 BT fractions (5.0-6.0 Gy) & & 2 & 14 \\
\hline \multirow[t]{2}{*}{5 BT fractions (5.5-6.0 Gy) } & & 7 & 50 \\
\hline & & Median & Range \\
\hline Patient age & & 58 & $33-79$ \\
\hline EBRT dose (Gy) & & 50 & $45-62$ \\
\hline BT dose (Gy) & & 26 & $16-30$ \\
\hline Plan characteristics & $N$ & Median & Range \\
\hline \multicolumn{4}{|l|}{ BT (plans) } \\
\hline Total fractions & 58 & & \\
\hline Usable fractions $^{\dagger}$ & 55 & & \\
\hline HR-CTV size (cc) & 32 & $8-145$ & \\
\hline Non-bulky (cc) & 15 & 15 & $8.3-20.0$ \\
\hline Low-bulky (cc) & 19 & 27 & $8.3-39.0$ \\
\hline Bulky (cc) & 21 & 74 & $8.3-145$ \\
\hline
\end{tabular}

FIGO - International Federation of Gynecology and Obstetrics, BT - brachytherapy, EBRT - external beam radiotherapy

${ }^{\dagger}$ For 3 fractions the MRI scanner was unavailable, and patients were imaged by $C T$ instead. These fractions were excluded from the study ingly used in diagnosis, its use in MRI-guided BT has only recently been introduced in the literature [12-15].

In this study, we retrospectively evaluated conventional plans using GEC-ESTRO recommended [5,16]. DVH parameters, and quantified the dosimetric benefits of MRIG-CBT, both in general and with respect to three different HR-CTV sizes.

\section{Material and methods}

\section{Patients and EBRT treatments}

We retrospectively studied 55 plans from 14 patients with biopsy-proven cervical cancer, with approval from the institutional review board. The malignancies were rated FIGO (International Federation of Gynecology and Obstetrics) stage Ib1 to IV and were treated in 2009 or 2010. The treatments employed EBRT with concomitant chemotherapy (for all but one patient in which chemotherapy was contraindicated), followed by 3-5 fractions of BT with 5.5-8 Gy fraction size. The institutional protocol specifies eight weeks from the beginning of EBRT to the end of BT; though this time was extended in five cases due to clinical complications. Other pertinent characteristics of the patients, tumors, and treatments are summarized in Table 1. EBRT treatment plans used either a 3-D conformal technique or intensity-modulated radiotherapy (IMRT) generated using the Pinnacle $3^{\circledR}$ treatment planning system (version 7.0, Philips Medical Systems Inc., Madison, WI, USA). The prescription dose of EBRT was 45 Gy in 25 fractions, and, when necessary, a 5.4 to $10 \mathrm{~Gy}$ boost in 3 to 5 fractions to the involved paraortic or parametrial regions.

\section{HDR treatments}

All 55 plans were treated using a conventional Point A planning technique. Intracavitary HDR started after 3-4 weeks of EBRT, using the ${ }^{192}$ Ir radioisotope (VariSource $\mathrm{i}{ }^{\circledR}{ }^{\circledR}$, Varian Medical Systems Inc., Palo Alto, CA, USA). Patients were prescribed a total dose of 33-36 $\mathrm{Gy}_{10}$ in 5-7 fractions, where $\mathrm{Gy}_{10}$ is the equivalent dose in $2 \mathrm{~Gy}$ fractions of EBRT (EQD2) using an $\alpha / \beta$ of $10 \mathrm{~Gy}$. For each fraction, clinical examination was performed under general anesthesia, and a FletcherSuit-Declos ${ }^{\circledR} \mathrm{T} \& \mathrm{O}$ applicator (Varian Medical Systems Inc.) was inserted under ultrasound guidance. A titanium applicator was chosen for advantages previously described in the literature $[12,17]$. Orthogonal digital radiographs were acquired before and after 3.0 Tesla MRI scan to identify applicator displacements during patient transport to the MR scanner (MAGNETOM TrioTM, Siemens Medical System Inc., Erlangen, Germany). All conventional plans were clinically generated on MRI not on radiographs, using a previously reported scan protocol [12]. T2- and T1-weighted MR images were used for contouring and source-pathway reconstruction, respectively.

\section{Conventional and MRIG-CBT HDR treatment planning}

Conventional point $\mathrm{A}$ based planning followed the institute's clinical protocol developed according to ABS guidelines [2] (Fig. 1). The point A was defined by drawing a line 


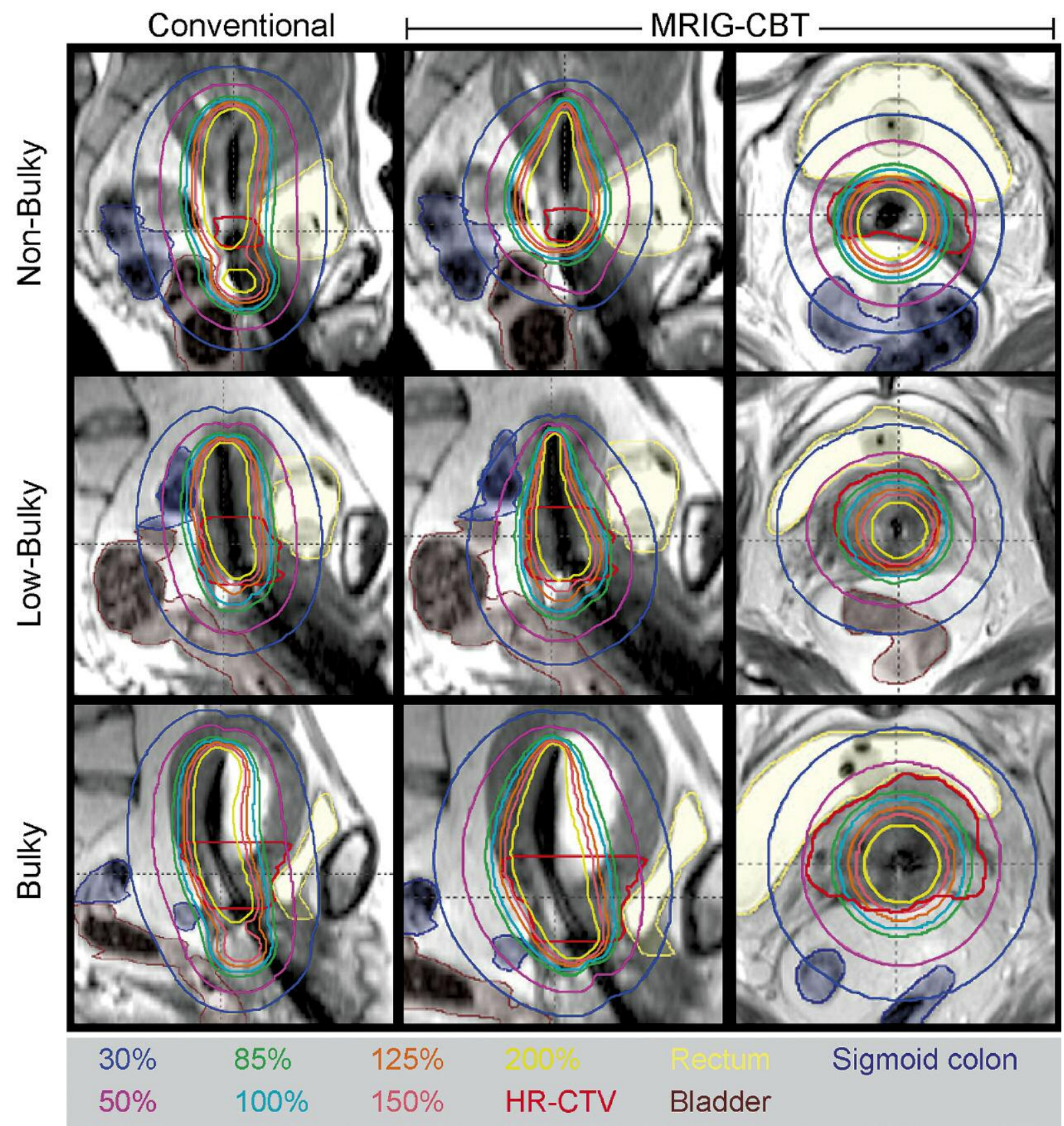

Fig. 1. Isodose lines for conventional and MRIG-CBT planning of all three HR-CTV sizes: non-bulky (HR-CTV < $20 \mathrm{cc}$ ), lowbulky $(20 \mathrm{cc} \leq \mathrm{HR}-\mathrm{CTV}<40 \mathrm{cc})$ and bulky $(\mathrm{HR}-\mathrm{CTV} \geq 40 \mathrm{cc})$

through the center of each ovoid, the radius of the ovoid + $2 \mathrm{~cm}$ superiorly along the tandem from the intersection of this line, and $2 \mathrm{~cm}$ lateral from the center of the tandem. Optimization lines were generated $0.5 \mathrm{~cm}$ lateral from each ovoid surface to specify vaginal doses, in addition to the lines of $2 \mathrm{~cm}$ lateral from the center of the tandem to specify point A doses. Source pathway reconstruction $[12,17]$ for T\&O was done on T1-weighted MRI (1 mm slice thickness) by importing predetermined shapes from the applicator library (SolidApplicator, BrachyVision ${ }^{\mathrm{TM}}$, Varian Medical System Inc.). According to the GEC-ESTRO recommendations
$[5,18]$, the HR-CTV, rectum, bladder and sigmoid colon were retrospectively contoured on T2-weighted MRI of $3 \mathrm{~mm}$ slice thickness.

The strategy for volume-optimization was based on a study by Kim et al. [12] (Fig. 1) using an hybrid-inverse planning process. Hybrid-inverse planning represents a 3-D volume-optimization plan not solely generated by inverse volume optimization, but generated in a hybrid manner using a conventional point A plan and inverse volumeoptimization. The first step is the generation of a conventional point A plan. A planner evaluates the DVH param- 
eters according to the total dose limit according to the GEC-ESTRO recommendations [19], the $\mathrm{D}_{2 \mathrm{cc}}$ of an OAR are $\leq 75 \mathrm{~Gy}_{3}$ of EQD2 for the rectum and sigmoid colon, and $\leq 90 \mathrm{~Gy}_{3}$ of EQD2 for the bladder. The dose-volume constraints in inverse-volume optimization are set so that all OARs receive equal or lower doses than their limit. Afterward the optimization is iteratively altered with updated dose-volume optimization constraints until three goals are achieved. The three goals are: 1) OAR $\mathrm{D}_{2 \mathrm{cc}}$ values below limits of equation (1) (defined below), 2) HR-CTV $\mathrm{D}_{90}$ below $105 \%$ of the prescription dose, and 3 ) if HR-CTV $D_{90}$ was below the prescription dose, at least one OAR $\mathrm{D}_{2 c c}$ value within $5 \%$ of its limit. The isodose lines for each slice on coronal, sagittal, and axial plans were reviewed, and final dose shaping using graphical optimization was performed if necessary.

As a "worst-case scenario", it was assumed that the HRCTV and all OARs received the EBRT prescription dose, and that each OAR $D_{2 c c}$ was in the same location for all BT treatment fractions [20]. The OAR $\mathrm{D}_{2 \mathrm{cc}}$ values from the EBRT boosts were also accounted for and used to determine an OAR limit for each BT fraction:

$$
L_{i}=\left(D_{L}-D_{E B R T}-D_{\text {boost }}\right) \frac{D_{i}}{D_{E T}}
$$

where $L_{i}$ is the $D_{2 c c}$ limit for BT fraction $\mathrm{i}, D_{L}$ is the total dose limit [19] to $\mathrm{D}_{2 \mathrm{cc}}$ of an OAR, $D_{E B R T}$ - the total EBRT dose, $D_{\text {boost }}$ - the $\mathrm{D}_{2 \mathrm{cc}}$ dose to the OAR from EBRT boost, $D_{i}$ is the prescribed $\mathrm{BT}$ dose for fraction $\mathrm{i}$, and $D_{B T}$ is the total prescribed BT dose. All OAR quantities were converted to EQD2 values using an $\alpha / \beta$ of 3 Gy [18].

\section{Analysis}

Each plan was analyzed by recording the $\mathrm{D}_{90}$ and $\mathrm{D}_{100}$ of the HR-CTV, the $\mathrm{D}_{2 \mathrm{cc}}$ and $\mathrm{D}_{0.1 \mathrm{cc}}$ of each OAR, and the doses to point $A$ and the ICRU bladder and rectum points in contrast to those with MRIG-CBT. It has been reported that DVH measurements in MRIG-CBT are highly-dependent on a number of variables, including applicator reconstruction, MRI artifacts, and contouring variation [17]. As both conventional and MRIG-CBT cases employ the same applicator location and contours, the comparison of the two methods was unaffected by these variables. All plans were divided into three volume categories: non-bulky (HR-CTV $<20 \mathrm{cc}$ ), low-bulky $(20 \mathrm{cc} \leq \mathrm{HR}-\mathrm{CTV}<40 \mathrm{cc}$ ) and bulky (HR$\mathrm{CTV} \geq 40 \mathrm{cc}$ ). The $p$ values were calculated using a two-tailed $t$-test assuming homoscedasticity.

\section{Results}

\section{Conventional, point A based plans}

All 55 conventional plans delivered the prescribed dose to point A with little variation (Table 2). The average doses to right and left point A were $100 \pm 1 \%$ (mean \pm standard deviation) of the respective prescription doses for non-bulky and low-bulky tumors. The plans for bulky tumors showed increased variation in point $A$ dose, at $100 \pm 2 \%$ of the respective prescription doses. The $\mathrm{D}_{90}$ of the HR-CTV for conventional plans was considerably affected by the HR-CTV volume. On average, $\mathrm{D}_{90}$ for the HR-CTV for the low-bulky tumors was favorable, at $104 \pm 17 \%$ (Table 2). However, we found the HR-CTV $\mathrm{D}_{90}$ for $47 \%$ of the low-bulky tumor plans corresponded to more than $5 \%$ overdose, while $42 \%$ of the plans received more than $5 \%$ under-dose (Table 3 ). For

Table 2. Conventional plan results

A: Conventional plan results, target coverage

\begin{tabular}{|c|c|c|c|c|c|}
\hline Target (\% Rx) & Pt. A dose & HR-CTV D 90 & Diff. & & \\
\hline All plans & $100 \pm 1$ & $93 \pm 24$ & $-7.1 \%$ & & \\
\hline Non-bulky & $100 \pm 1$ & $111 \pm 16$ & $10.6 \%$ & & \\
\hline Low-bulky & $100 \pm 1$ & $103 \pm 16$ & $3.0 \%$ & & \\
\hline Bulky & $100 \pm 2$ & $72 \pm 17$ & $-28.8 \%$ & & \\
\hline \multicolumn{6}{|c|}{ B: Conventional plan results, OAR sparring } \\
\hline & ICRU (Gy) Pt. & $D_{0.1 c c}(G y)$ & $\mathrm{D}_{0.1 c c} / \mathrm{D}_{\text {ICRU }}$ & $D_{2 c c}(G y)$ & $\mathrm{D}_{2 \mathrm{cc}} / \mathrm{D}_{\mathrm{ICRU}}$ \\
\hline \multicolumn{6}{|l|}{ Rectum } \\
\hline All plans & $3.9 \pm 1.5$ & $5.0 \pm 1.7$ & $1.3 \pm 0.4$ & $3.7 \pm 1.1$ & $1.0 \pm 0.3$ \\
\hline Non-bulky & $4.2 \pm 1.8$ & $5.1 \pm 1.7$ & $1.4 \pm 0.5$ & $3.8 \pm 1.3$ & $1.0 \pm 0.4$ \\
\hline Low-bulky & $3.9 \pm 1.6$ & $5.0 \pm 2.0$ & $1.3 \pm 0.3$ & $3.7 \pm 1.3$ & $1.0 \pm 0.3$ \\
\hline Bulky & $3.8 \pm 1.1$ & $4.9 \pm 1.5$ & $1.3 \pm 0.5$ & $3.7 \pm 0.9$ & $1.0 \pm 0.3$ \\
\hline \multicolumn{6}{|l|}{ Bladder (\% Lim) } \\
\hline All plans & $4.5 \pm 1.8$ & $6.7 \pm 2.2$ & $1.7 \pm 0.8$ & $5.1 \pm 1.4$ & $1.3 \pm 0.6$ \\
\hline Non-bulky & $3.8 \pm 1.7$ & $5.9 \pm 1.7$ & $1.7 \pm 0.4$ & $4.6 \pm 1.5$ & $1.3 \pm 0.3$ \\
\hline Low-bulky & $4.6 \pm 1.9$ & $7.0 \pm 1.8$ & $1.8 \pm 0.9$ & $5.3 \pm 0.9$ & $1.4 \pm 0.7$ \\
\hline Bulky & $4.7 \pm 1.7$ & $7.1 \pm 2.8$ & $1.7 \pm 0.9$ & $5.4 \pm 1.6$ & $1.3 \pm 0.7$ \\
\hline
\end{tabular}

$R x$ - prescribed dose, Pt - point, HR-CTV - high-risk clinical target volume, $D_{90}$-minimum dose to the hottest $90 \%$ of the target volume, OAR-organ-at-risk, ICRU Pt - point recommended by ICRU report \#38 [1], D ICC $D_{2 C C}$ - minimum dose to the hottest $0.1 \mathrm{cC}$ or $2 \mathrm{cC}$ of the OAR volume, DICRU - dose to an ICRU Point. All values are mean \pm one standard deviation 
non-bulky or bulky tumors, the average HR-CTV $\mathrm{D}_{90}$ values were either considerable overdoses $(+10.5 \%)$ or underdoses $(-28.2 \%)$, respectively. Regardless of their tumor size, only $16 \%$ of conventional plans had HR-CTV $\mathrm{D}_{90}$ values within $5 \%$ of the prescription dose (Table 3 ). Of the conventional plans, $52.7 \%$, had at least one OAR with a $\mathrm{D}_{2 \mathrm{cc}}$ value above the GEC-ESTRO limit. The $\mathrm{D}_{2 \mathrm{cc}}$ limits for the rectum, bladder, and sigmoid colon were exceeded in $20 \%, 29 \%$, and $18 \%$ of the conventional plans, respectively (Table 3 ). The bladder was the most frequently overdosed OAR ( $29 \%$ of plans, Table 3), despite having a higher recommended dose limit than the rectum or sigmoid colon. The mean doses of each OAR across all plans remained below limits for all HR-CTV sizes. When normalized to the fraction $\mathrm{D}_{2 \mathrm{cc}}$ limits of equation 1 , mean OAR $\mathrm{D}_{2 \mathrm{cc}}$ ranged from $66 \pm 21 \%$ for the sigmoid colon in non-bulky geometries to a high of $94 \pm 34 \%$ for bladder near low-bulky HR-CTVs (Table 4). When the dose distributions for all HDR and EBRT fractions were considered cumulatively, four of the twelve patients for which all HDR fractions were available showed at least one OAR $\mathrm{D}_{2 \mathrm{cc}}$ value over the limit for the entire treatment: one patient had $D_{2 c c}$ values over the limit for both rectum and bladder, one patient exceeded only the rectum limit, one only the bladder limit, and one only the limit of the sigmoid colon.

Statistically significant correlation was found between the rectum ICRU point and its $\mathrm{D}_{2 \mathrm{cc}}$ measurements $\left(r^{2}=0.5449\right.$, $p<0.001$ ) (Table 2). The mean ratio of $\mathrm{D}_{2 \mathrm{cc}}$ to $\mathrm{D}_{\text {ICRU }}$ for the rectum was $1.0 \pm 0.3$. However, no significant correlation was seen between the bladder ICRU point and $\mathrm{D}_{2 \mathrm{cc}}\left(r^{2}=0.210\right.$, $p=0.125)$. The bladder ICRU point underestimated maximal dose $\left(D_{2 c c}\right)$, i.e. the mean ratio of $D_{2 c C}$ to $D_{\text {ICRU }}$ was $1.3 \pm 0.6$.

\section{MRIG-CBT plans}

MRIG-CBT plans exhibited significantly higher HR-CTV $\mathrm{D}_{90}$ values for non-bulky $(p=0.002)$ and bulky tumors $(p=0.028)$ (Table 4$)$. No MRIG-CBT plans had a $\mathrm{D}_{90}$ greater than $5 \%$ above the prescription dose. No MRIG-CBT plans exhibited $\mathrm{D}_{2 \mathrm{cc}}$ of any OARs over their GEC-ESTRO limit (Table 3). Thus, no patients showed overall $\mathrm{D}_{2 c c}$ values over the limits throughout their full HDR treatments. The effect of this restriction was seen in the HR-CTV coverage result: for all plans where HR-CTV $\mathrm{D}_{90}$ was less than the prescription dose, at one or more OAR $\mathrm{D}_{2 \mathrm{cc}}$ was within $5 \%$ of its limit: this OAR was the bladder in $96 \%$ of these plans, the rectum in $20 \%$, and the sigmoid colon in $20 \%$. The average $D_{2 c c}$ values of MRIG-CBT plans ranged from $41 \pm 16 \%$ of the fraction limit for rectum of non-bulky cases and $87 \pm 16 \%$ for bladder in bulky plans (Table 4). The bladder was the only structure limiting dose in non-bulky and low-bulky plans. For the 16 bulky plans with HR-CTV $\mathrm{D}_{90}$ less than the prescribed dose, the bladder $\mathrm{D}_{2 \mathrm{cc}}$ was within $5 \%$ of its limit in $81 \%$ of the plans, and the rectum and sigmoid $D_{2 c c}$ were each dose-restricting in $31 \%$ of such cases.

To visually compare volumetric measurements (Fig. 2), the mean success of each plan attribute was plotted on a separate axis; HR-CTV $\mathrm{D}_{90}$ is plotted on the vertical axis relative to the prescription dose $(\mathrm{Rx})$. Therefore, the $100 \%$ val-

Table 3. Conventional and MRIG-CBT plans. Analysis with respect to tumor coverage and organs-at-risk sparing

\begin{tabular}{|c|c|c|c|c|c|c|c|c|}
\hline \multirow[t]{2}{*}{ Plan characteristics } & \multicolumn{2}{|c|}{ All sizes (\%) } & \multicolumn{2}{|c|}{ Non-bulky (\%) } & \multicolumn{2}{|c|}{ Low-bulky (\%) } & \multicolumn{2}{|c|}{ Bulky (\%) } \\
\hline & Conv. & MRIG. & Conv. & MRIG. & Conv. & MRIG. & Conv. & MRIG. \\
\hline \multicolumn{9}{|l|}{ Point A } \\
\hline Over $\mathrm{Rx}+5 \%$ & 2 & 35 & 0 & 13 & 0 & 37 & 5 & 48 \\
\hline Within $\mathrm{Rx} \pm 5 \%$ & 98 & 16 & 100 & 13 & 100 & 11 & 95 & 24 \\
\hline Under Rx $-5 \%$ & 0 & 49 & 0 & 73 & 0 & 53 & 0 & 29 \\
\hline \multicolumn{9}{|c|}{ ICRU point over $80 \%$ Rx } \\
\hline Rectum ICRU & 22 & 22 & 33 & 13 & 16 & 21 & 19 & 29 \\
\hline Bladder ICRU & 42 & 47 & 27 & 13 & 58 & 47 & 38 & 71 \\
\hline \multicolumn{9}{|l|}{ HR-CTV $D_{90}$} \\
\hline Over $\mathrm{Rx}+5 \%$ & 33 & 0 & 53 & 0 & 47 & 0 & 5 & 0 \\
\hline Within $\mathrm{Rx} \pm 5 \%$ & 16 & 56 & 33 & 87 & 11 & 68 & 10 & 24 \\
\hline Under Rx $-5 \%$ & 51 & 44 & 13 & 13 & 42 & 32 & 86 & 76 \\
\hline \multicolumn{9}{|l|}{$\mathrm{D}_{2 c c}$ over OAR limit } \\
\hline Rectum $D_{2 c c}$ & 20 & 0 & 13 & 0 & 26 & 0 & 19 & 0 \\
\hline Bladder $\mathrm{D}_{2 \mathrm{cc}}$ & 29 & 0 & 13 & 0 & 37 & 0 & 33 & 0 \\
\hline Sigmoid $D_{2 c c}$ & 18 & 0 & 7 & 0 & 5 & 0 & 38 & 0 \\
\hline \multicolumn{9}{|c|}{$\mathrm{D}_{2 c c}$ within $\pm 5 \%$ of OAR limit } \\
\hline Rectum $D_{2 c c}$ & 7 & 9 & 7 & 0 & 0 & 0 & 14 & 24 \\
\hline Bladder $\mathrm{D}_{2 \mathrm{cc}}$ & 13 & 44 & 20 & 13 & 5 & 47 & 14 & 62 \\
\hline Sigmoid $D_{2 c c}$ & 11 & 9 & 13 & 0 & 11 & 0 & 10 & 24 \\
\hline
\end{tabular}

OAR - organ-at-risk, $D_{2 c c}, D_{0.1 c c}$ - minimum dose to the hottest $0.1 \mathrm{cc}$ or $2 \mathrm{cc}$ of the OAR volume, Rx-prescribed dose, HR-CTV-high-risk clinical target volume, $D_{90}$ - minimum dose to the hottest $90 \%$ of the volume 


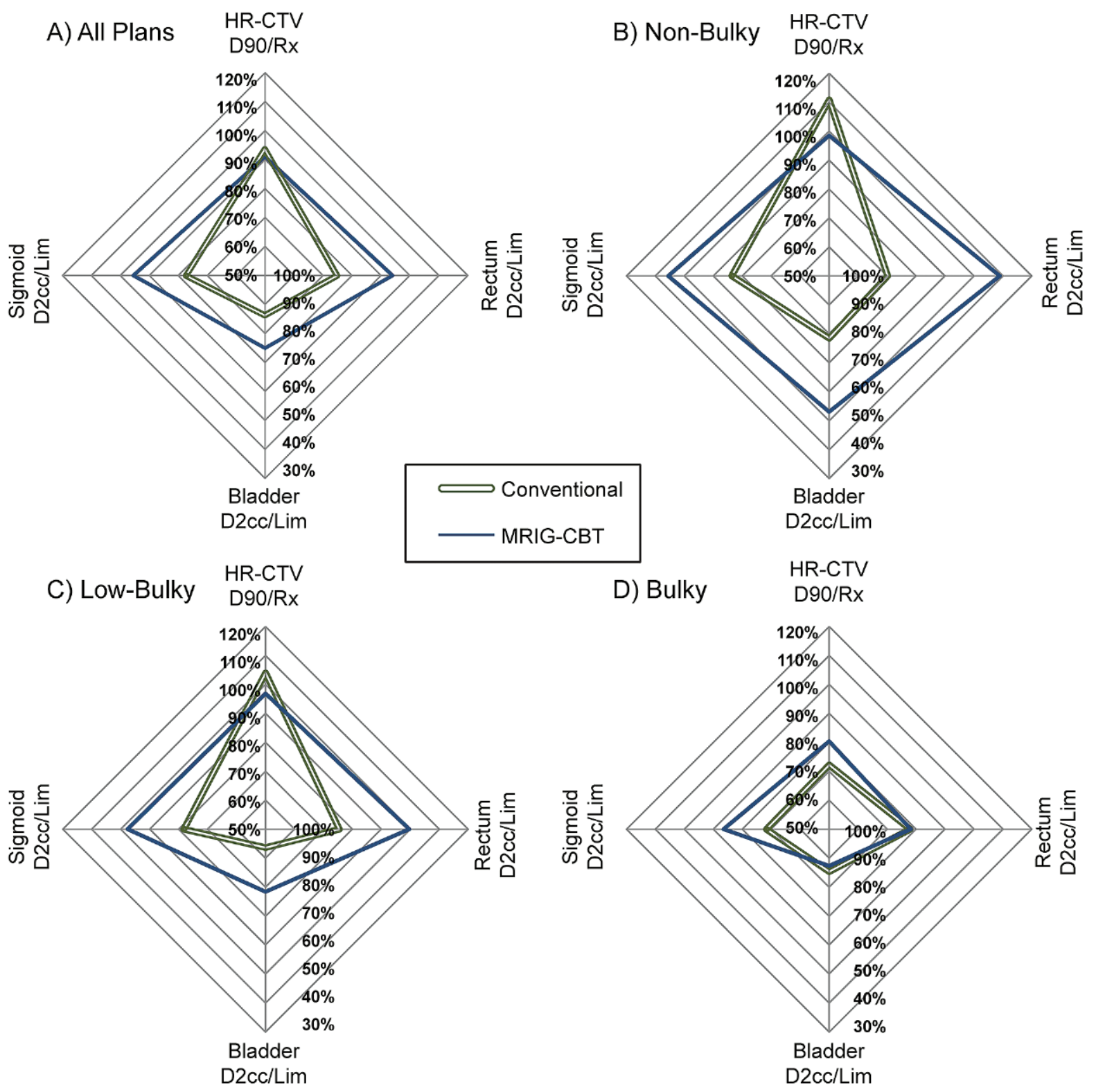

Fig. 2. Average plan attributes for each HR-CTV size. HR-CTV $D_{90}$ is plotted on the vertical axis relative to the prescription dose. Therefore, the $100 \%$ of HR-CTV $\mathrm{D}_{90}$ / prescription dose (Rx) represents HR-CTV $\mathrm{D}_{90}$ receives $\mathrm{Rx}$. The $\mathrm{D}_{2 \mathrm{cc}}$ of each OAR relative to the OAR limit $\left(\mathrm{D}_{2 c \mathrm{c}} / \mathrm{Lim}\right)$ is plotted on the other three axes, thus smaller \% values represent better OAR sparing; these axes are inverted such that OAR sparing increased as the values of sigmoid, bladder, rectum move away from the origin

ue of HR-CTV $\mathrm{D}_{90}$ / prescription dose (HR-CTV $\left.\mathrm{D}_{90} / \mathrm{Rx}\right)$ on the vertical axis is ideal. For instance, in B panel, conventional plans have $111 \%$ Rx at HR-CTV $D_{90}$, while MRIG-CBT plans deliver $99 \% \mathrm{Rx}$. The $\mathrm{D}_{2 \mathrm{cc}}$ of each OAR relative to the OAR limit $\left(\mathrm{D}_{2 \mathrm{cc}} / \mathrm{Lim}\right)$ is plotted on the other three axes, thus smaller \% values represent better OAR sparing; these axes were inverted such that OAR sparing increased as the values of sigmoid, bladder, rectum move away from the origin. Thus a larger area of the plotted "diamonds" indicates improved plan characteristics. Large improvements were seen for both low- and non-bulky cases, while mean treatment results of bulky HR-CTV plans remained comparable (Table 3).

\section{Discussion}

The dosimetric improvements of MRIG-CBT plans have, when compared to conventional plans, been published for HDR [19], and PDR [6-9] BT when using tandem-and-ring applicator $(\mathrm{T} \& \mathrm{R})[7,9], \mathrm{T} \& \mathrm{R}$ plus needles [7,9], T\&O [6], or tandem only [8]. However, previous studies analyzed the benefits of MRIG-CBT without tumor size classification or with only two tumor size classifications, large and small volumes. In this study, the dosimetric benefits of MRIG-CBT were analyzed for three different HR-CTV groups when using conventional $\mathrm{T} \& \mathrm{O}$ applicators without the use of supplementary needles. For bulky tumors, however, neither 
Table 4. MRIG-CBT plan results

A: Conventional plan results, target coverage

\begin{tabular}{|c|c|c|c|c|c|c|c|c|}
\hline HR-CTV D 90 & $\begin{array}{l}\text { Conventional } \\
(\% R x)\end{array}$ & $\begin{array}{c}\text { MRIG-CBT } \\
(\% \mathrm{Rx})\end{array}$ & Diff. (\%) & $p$-value & & & & \\
\hline All plans & $93 \pm 24$ & $91 \pm 15$ & -7.6 & 0.421 & & & & \\
\hline Non-bulky & $111 \pm 16$ & $98 \pm 9$ & -9.2 & 0.002 & & & & \\
\hline Low-bulky & $103 \pm 16$ & $97 \pm 9$ & -6.2 & 0.101 & & & & \\
\hline Bulky & $72 \pm 17$ & $80 \pm 16$ & -7.7 & 0.028 & & & & \\
\hline \multicolumn{9}{|c|}{ B: Planning comparison, OAR sparring } \\
\hline & \multicolumn{3}{|c|}{ Conventional } & \multicolumn{3}{|c|}{ MRIG-CBT } & Diff. of $\%$ & $p$-value \\
\hline & $D_{0.1 c c}(G y)$ & $\mathrm{D}_{2 c c}(\mathrm{~Gy})$ & $\%$ OAR limit & $\mathrm{D}_{0.1 \mathrm{cc}}(\mathrm{Gy})$ & $D_{2 c c}(G y)$ & $\%$ OAR limit & OAR limits & \\
\hline \multicolumn{9}{|l|}{ Rectum } \\
\hline All plans & $5.0 \pm 1.7$ & $3.7 \pm 1.1$ & $75 \pm 34$ & $4.2 \pm 1.4$ & $3.1 \pm 1.0$ & $56 \pm 25$ & $-19.0 \%$ & $<0.001$ \\
\hline Non-bulky & $5.1 \pm 1.7$ & $3.8 \pm 1.3$ & $80 \pm 43$ & $3.6 \pm 1.2$ & $2.5 \pm 0.7$ & $41 \pm 16$ & $-38.7 \%$ & $<0.001$ \\
\hline Low-bulky & $5.0 \pm 2.0$ & $3.7 \pm 1.3$ & $74 \pm 32$ & $3.9 \pm 1.3$ & $2.9 \pm 0.8$ & $50 \pm 19$ & $-24.1 \%$ & $<0.001$ \\
\hline Bulky & $4.9 \pm 1.5$ & $3.7 \pm 0.9$ & $72 \pm 29$ & $4.9 \pm 1.4$ & $3.7 \pm 1.0$ & $72 \pm 26$ & $-0.1 \%$ & 0.893 \\
\hline \multicolumn{9}{|l|}{ Bladder } \\
\hline All plans & $6.7 \pm 2.2$ & $5.1 \pm 1.4$ & $86 \pm 36$ & $6.3 \pm 1.7$ & $4.7 \pm 1.3$ & $75 \pm 25$ & $-11.4 \%$ & 0.006 \\
\hline Non-bulky & $5.9 \pm 1.7$ & $4.6 \pm 1.5$ & $78 \pm 40$ & $4.9 \pm 1.6$ & $3.6 \pm 1.2$ & $53 \pm 28$ & $-25.4 \%$ & $<0.001$ \\
\hline Low-bulky & $7.0 \pm 1.8$ & $5.3 \pm 0.9$ & $94 \pm 34$ & $6.5 \pm 1.3$ & $4.8 \pm 0.9$ & $78 \pm 21$ & $-15.2 \%$ & 0.009 \\
\hline Bulky & $7.1 \pm 2.8$ & $5.4 \pm 1.6$ & $85 \pm 34$ & $7.1 \pm 1.6$ & $5.5 \pm 1.0$ & $87 \pm 16$ & $2 \%$ & 0.502 \\
\hline \multicolumn{9}{|l|}{ Sigmoid } \\
\hline All plans & $5.1 \pm 2.2$ & $3.6 \pm 1.1$ & $73 \pm 31$ & $4.3 \pm 1.9$ & $3.0 \pm 0.9$ & $54 \pm 23$ & $-18.4 \%$ & $<0.001$ \\
\hline Non-bulky & $4.8 \pm 1.4$ & $3.5 \pm 1.0$ & $66 \pm 21$ & $4.0 \pm 1.6$ & $2.7 \pm 0.9$ & $44 \pm 14$ & $-22.0 \%$ & $<0.001$ \\
\hline Low-bulky & $4.7 \pm 1.4$ & $3.5 \pm 1.1$ & $72 \pm 30$ & $3.9 \pm 1.3$ & $2.9 \pm 0.8$ & $52 \pm 21$ & $-19.4 \%$ & $<0.001$ \\
\hline Bulky & $5.6 \pm 3.1$ & $3.8 \pm 1.3$ & $78 \pm 38$ & $4.9 \pm 2.4$ & $3.4 \pm 1.0$ & $64 \pm 28$ & $-14.8 \%$ & 0.006 \\
\hline
\end{tabular}

OAR - organ-at-risk, $D_{2 c c}, D_{0.1 c c}$ - minimum dose to the hottest $0.1 \mathrm{cc}$ or $2 \mathrm{cc}$ of the OAR volume, Rx-prescribed dose, HR-CTV - high-risk clinical target volume, $D_{90}$ - minimum dose to the hottest $90 \%$ of the volume

planning method was able to increase HR-CTV $\mathrm{D}_{90}$ to the prescribed dose when the T\&O was used. MRIG-CBT resulted in only small improvements for bulky tumors. Therefore, the only use of conformal volume optimization and MRI guidance are limited to appropriately cover bulky tumors when a conventional intracavitary applicator is used. MRIG-CBT applicators for bulky tumors have been developed: T\&R plus needles (Vienna applicator) $[7,9]$ and $T \& O$ plus needles (Utrecht applicator) [21] and their use is recommended for bulky tumors. Transperineal interstitial implants for MRIG-CBT can be an alternative technique for bulky tumors. As a less invasive approach for bulky tumors, intensity-modulated brachytherapy using intracavitary applicators with electronic brachytherapy source has been under development [22,23].

The MRIG-CBT treatment planning approach showed good HR-CTV $\mathrm{D}_{90}$ in all cases where it was not restricted by OAR dose limits. Most of the optimization results were highly dependent on the OAR $\mathrm{D}_{2 \mathrm{cc}}$ limits set by equation (1). In cases where the HR-CTV $D_{90}$ did not reach the prescription dose, one of the three OARs restricted the HR-CTV $\mathrm{D}_{90}$, and increasing the limit or allowing a slight overdose could have a large effect on HR-CTV $D_{90}$. This may be pos- sible by shifting more of the allotted OAR dose to earlier HDR fractions and relying on tumor regression to enable sparing in the corresponding OAR in later treatment fractions. Such a shift in OAR limits would also affect the relative results of the two planning methods. In the present study, the optimization was able to reduce systematic overdoses to the HR-CTV in non-bulky plans, and reduce the $\mathrm{D}_{90}$ variance seen in the conventional coverage of low-bulky tumors. Especially, for non-bulky tumors, MRIG-CBT can result in the dose reduction on side wall and nodes. Future studies on nodal doses of MRIG-CBT need to be performed. The studies on how to deliver appropriate radiation dose to nodes through EBRT also are expected to be followed. For instance, a simultaneous boosting to pelvic lymph nodes can be an option using image guided IMRT technique.

As emphasized by Trnková et al. [3], large-scale modifications to conventional distributions and/or solely inverse planning should be avoided until an understanding of the relationship between DVH-values and treatment efficacy is established. Thus, hybrid inverse optimization [24] was utilized in this study. What constitutes a "large-scale" modification and how to find improved inverse planning objectives are still open questions. 
A low-magnetic-field MR system of 1.5 or 2.0 Tesla has been used for MR-guided BT. However, the signal-to-noise ratio (SNR) of a 1.5 Tesla MR is suboptimal, particularly its ability to accurately delineate the uterine cervix and the macroscopic tumor regions $[14,24]$. In addition, increased intra-scan motion artifacts exist when using 1.5 Tesla MR due to the extended acquisition time when compared to a 3 Tesla MR (see Fig. 3 in reference [11]). The high resolution (3 Tesla) MR system used in this study has approximately double the SNR of a 1.5 Tesla system, resulting in an image with a higher contrast in the uterine cervix and vagina [11] and a substantially faster image acquisition speed $[12,25]$. However, caution must be exercised when metal based applicators are used in 3 Tesla MRI-guided BT scans. In this study, a titanium T\&O applicator was used with a 3 Tesla MRI. Before any clinical use of 3 Tesla MRIguided BT, the safety concerns, especially radiofrequency $[R F]$ heat- had been verified first [26]. Once assured of the safety of the process, the artifacts and distortions of the titanium applicator were quantified [12], since the artifact to noise ratio also increases in 3 Tesla MR systems.

Although it has been suggested to replace the BT component of cervical cancer treatment with stereotactic body RT, the unique advantages of BT should not be overlooked. BT offers a significantly higher biologically-effective dose due to the short distance between radiation sources and target volumes, and the sharp fall-off in radiation dose in space allows for higher dose to non-bulky and low-bulky tumor volumes while still retaining adequate sparing of nearby healthy tissues.

\section{Conclusions}

HR-CTV $D_{90}$ was considerably affected by tumor size. Only $16 \%$ of conventional plans had HR-CTV $D_{90}$ values within $5 \%$ of the prescription dose. Mean $\mathrm{D}_{2 \mathrm{cc}}$ values of rectum, bladder, and sigmoid colon were found to be below the GEC-ESTRO recommended limits for the conventional plans. However, in $52.7 \%$ of conventional plans at least one of the OARs had a $\mathrm{D}_{2 \mathrm{cc}}$ value over its limit. When all HDR fractions were considered cumulatively, $17 \%$ of patients had overall rectum and bladder $\mathrm{D}_{2 \mathrm{cc}}$ values over their limits, while $8 \%$ of patients exceeded the limit for sigmoid colon. The MRIG-CBT treatment planning technique was found to improve treatment plans relative to those of the conventional approach with HR-CTV less that $40 \mathrm{cc}$, bringing the $\mathrm{D}_{90}$ dose closer to the prescription value while avoiding overdose to the $\mathrm{D}_{2 \mathrm{cc}}$ of each OAR. Using conventional intracavitary applicators, only small improvements were seen when MRIGCBT was used for bulky targets (> $40 \mathrm{cc}$ ).

\section{References}

1. "ICRU-report-38". Dose and volume specification for reporting intracavitary therapy in gynecology. International Commission on Radiation Units and Measurements, Bethesda 1985.

2. Viswanathan AN, Thomadsen B, Committee ABSCCR. American Brachytherapy Society consensus guidelines for locally advanced carcinoma of the cervix. Part I: general principles. Brachytherapy 2012; 11: 33-46.

3. Trnkova P, Pötter R, Baltas D et al. New inverse planning technology for image-guided cervical cancer brachytherapy: description and evaluation within a clinical frame. Radiother Oncol 2009; 93: $331-340$
4. Viswanathan AN, Dimopoulos J, Kirisits C et al. Computed tomography versus magnetic resonance imaging-based contouring in cervical cancer brachytherapy: results of a prospective trial and preliminary guidelines for standardized contours. Int J Radiat Oncol Biol Phys 2007; 68: 491-498.

5. Haie-Meder C, Pötter R, Van Limbergen E et al. Recommendations from gynaecological (GYN) GEC-ESTRO Working Group (I): concepts and terms in 3D image based 3D treatment planning in cervix cancer brachytherapy with emphasis on MRI assessment of GTV and CTV. Radiother Oncol 2005; 74: 235-245.

6. De Brabandere M, Mousa AG, Nulens A et al. Potential of dose optimisation in MRI-based PDR brachytherapy of cervix carcinoma. Radiother Oncol 2008; 88: 217-226.

7. Lindegaard JC, Tanderup K, Nielsen SK et al. MRI-guided 3D optimization significantly improves DVH parameters of pulsed-dose-rate brachytherapy in locally advanced cervical cancer. Int J Radiat Oncol Biol Phys 2008; 71: 756-764.

8. Zwahlen D, Jezioranski J, Chan P et al. Magnetic resonance imaging-guided intracavitary brachytherapy for cancer of the cervix. Int J Radiat Oncol Biol Phys 2009; 74: 1157-1164.

9. Tanderup K, Nielsen SK, Nyvang GB et al. From point A to the sculpted pear: MR image guidance significantly improves tumour dose and sparing of organs at risk in brachytherapy of cervical cancer. Radiother Oncol 2010; 94: 173-180.

10. Dimopoulos JC, Lang S, Kirisits C et al. Dose-volume histogram parameters and local tumor control in magnetic resonance image-guided cervical cancer brachytherapy. Int J Radiat Oncol Biol Phys 2009; 75: 56-63.

11. Kataoka M, Kido A, Koyama T et al. MRI of the female pelvis at 3T compared to 1.5T: evaluation on high-resolution T2-weighted and HASTE images. J Magn Reson Imaging 2007; 25: 527-534.

12. Kim Y, Muruganandham M, Modrick JM et al. Evaluation of artifacts and distortions of titanium applicators on 3.0-Tesla MRI: feasibility of titanium applicators in MRI-guided brachytherapy for gynecological cancer. Int $\mathrm{J}$ Radiat Oncol Biol Phys 2011; 80: 947-955.

13. Anderson J, Huang Y, Kim Y. Dosimetric impact of point A definition on high-dose-rate brachytherapy for cervical cancer: evaluations on conventional point A and MRI-guided, conformal plans. J Contemp Brachytherapy 2012; 4: 241-246.

14. Kapur T, Egger J, Damato A et al. 3-T MR-guided brachytherapy for gynecologic malignancies. Magn Reson Imag 2012; 30: 1279-1290.

15. Bubley GJ, Bloch BN, Vazquez C et al. Accuracy of endorectal magnetic resonance/transrectal ultrasound fusion for detection of prostate cancer during brachytherapy. Urology 2013; 81: 1284-1290.

16. Hellebust TP, Kirisits C, Berger D et al. Recommendations from gynaecological (GYN) GEC-ESTRO working group: considerations and pitfalls in commissioning and applicator reconstruction in $3 \mathrm{D}$ image-based treatment planning of cervix cancer brachytherapy. Radiother Oncol 2010; 96: 153-160.

17. Haack S, Nielsen SK, Lindegaard JC et al. Applicator reconstruction in MRI 3D image-based dose planning of brachytherapy for cervical cancer. Radiother Oncol 2009; 91: 187-193.

18. Pötter R, Haie-Meder C, Van Limbergen E et al. Recommendations from gynaecological (GYN) GEC ESTRO working group (II): concepts and terms in 3D image-based treatment planning in cervix cancer brachytherapy-3D dose volume parameters and aspects of 3D image-based anatomy, radiation physics, radiobiology. Radiother Oncol 2006; 78: 67-77.

19. Kirisits C, Pötter R, Lang $S$ et al. Dose and volume parameters for MRI-based treatment planning in intracavitary brachytherapy for cervical cancer. Int J Radiat Oncol Biol Phys 2005; 62: 901-911.

20. Kirisits C, Pötter R, Lang S et al. Dose and volume parameters for MRI-based treatment planning in intracavitary brachyther- 
apy for cervical cancer. Int J Radiat Oncol Biol Phys 2005; 62: 901-911.

21. Jurgenliemk-Schulz IM, Tersteeg RJ, Roesink JM et al. MRIguided treatment-planning optimisation in intracavitary or combined intracavitary/interstitial PDR brachytherapy using tandem ovoid applicators in locally advanced cervical cancer. Radiother Oncol 2009; 93: 322-330.

22. Yang W, Kim $Y$, Wu $X$ et al. Rotating-shield brachytherapy for cervical cancer. Phys Med Biol 2013; 58: 3931-3941.

23. Liu Y, Flynn RT, Yang W et al. Rapid emission angle selection for rotating-shield brachytherapy. Med Phys 2013; 40: 051720.

24. Kim Y, Modrick JM, Bayouth JE et al. Logistics of tandem and ovoids HDR plan optimization in MRI-guided brachytherapy for cervical cancer: comparisons of standard, graphical, and inverse optimization. Brachytherapy 2009; 8: 121-122.

25. Sheu MH, Chang CY, Wang JH et al. Preoperative staging of cervical carcinoma with MR imaging: a reappraisal of diagnostic accuracy and pitfalls. Eur Radiol 2001; 11: 1828-1833.

26. Hussain SM, van den Bos IC, Oliveto JM et al. MR imaging of the female pelvis at 3T. Magn Reson Imaging Clin N Am 2006; 14: 537-544, vii.

27. Nixon E, Kim Y, Kearney WR et al. HDR brachytherapy tandem and ovoid titanium applicator safety assessment in $3 \mathrm{~T}$ MRI. Brachytherapy 2008; 7: 135. 